\title{
COMPUTING WITH HECKE GRÖSSENCHARACTERS
}

\author{
by
}

\author{
Mark Watkins
}

\begin{abstract}
We describe how to compute with algebraic Hecke Grössencharacters. We first describe Dirichlet characters on number field elements, and finite order Hecke characters on ideals, before passing to the general case. Our primary interest is not directly in the characters themselves, but rather in their $L$-functions, and particularly the special values of these. We avoid the adelic language, since it does not readily lend itself to computer implementation. We give many numerical examples that have been computed with the Magma computer algebra system.

Résumé. - Nous montrons comment il est possible d'effectuer des calculs en utilisant les "Grössencharacters". Dans un premier temps, on décrit les caractères de Dirichlet des corps de nombres ainsi que les caractères de Hecke d'ordre fini sur les idéaux, avant de passer au cas général. Notre principal intérêt n'est pas directement les caractères pour eux-mêmes, mais plutôt les fonctions $L$ associées, en particulier leurs valeurs spéciales. Nous évitons le langage adélique puisqu'il n'est pas propre à l'implémentation directe sur machine. Nous donnons de nombreux exemples numériques calculés avec le système de calcul formel Magma.
\end{abstract}

\section{Introduction}

We give a description of how to compute with algebraic Hecke Grössencharacters. Due to the computational nature of our undertaking, we try to avoid the adelic language as much as possible, choosing a more explicit phrasing. We start by reviewing Dirichlet characters (on field elements) and finite order Hecke characters (on ideals), before passing to the Grössencharacter case. The algorithmic problems of interest include discrete logarithms for residue and ray class groups (see [12]), and principalisation of ideals when Grössencharacters are introduced. We also need to be careful about embeddings and choices of extension fields at various places. Convenient references are Tate's thesis [23] (though it uses the adelic language), and Chapter Zero of Schappacher's book [19].

Key words and phrases. - Hecke Grössencharacters, ray class groups, L-functions, special values.

This paper grew out of a talk given at a CIRM meeting in Luminy in Nov-Dec 2009 on Théorie des nombres et applications, and the author thanks the organisers for the invitation. An implementation is contained in version 2.16 of Magma [2]. Thanks also to N. P. Dummigan for sorting out some details in Example 6.1. 
Our end goal is not only to compute with the characters themselves, but also with their $L$-functions. The work of Lavrik [14] generalises that of Hecke, and allows us to approximate any value of an $L$-function via a "rapidly converging" series, which in practise means that we need to take about $\sqrt{N}$ terms of the $L$-series, where $N$ is the conductor. We use the Magma [2] implementation of Dokchitser [6]. As an example, computing the special values $L(\mathbf{G}, 2)$ in $\S 6.1$ to hundreds of digits takes only a few seconds.

1.1. Notation. - We let $\zeta_{k}$ denote an unspecified primitive $k$ th root of unity, and write $\mathrm{Na}$ for the norm of an ideal $\mathfrak{a}$. We will often write (say) an expression like $\mathfrak{p}_{2}=(1+i)=\left(e_{2}\right)$, and take this to define $e_{2}=1+i$. A tensor product with no subscript will be taken to be over $\mathbf{Q}$.

\section{Dirichlet characters}

Let $K$ be a number field, and let $\mathcal{I}$ be an ideal contained in its ring of integers $\mathbf{Z}_{K}$. Reduction modulo $\mathcal{I}$ yields a ring of residue classes corresponding to $\mathbf{Z}_{K} / \mathcal{I}$, and the Dirichlet character group modulo $\mathcal{I}$ is dual to the multiplicative group of units given by $\left(\mathbf{Z}_{K} / \mathcal{I}\right)^{\star}$. We can decompose this latter group over the prime power factors of $\mathcal{I}$ as

$$
\left(\mathbf{Z}_{K} / \mathcal{I}\right)^{\star} \cong \prod_{\mathfrak{p}^{k} \| \mathcal{I}}\left(\mathbf{Z}_{K} / \mathfrak{p}^{k}\right)^{\star}
$$

which will allow us to restrict and induce characters.

We let $\Omega_{K}^{\mathbf{R}}$ and $\Omega_{K}^{\mathbf{C}}$ be respectively the sets of real and complex infinite places of $K$. We can also include characters associated to ramification at real infinite places in $\Omega_{K}^{\mathbf{R}}$. Each real infinite place $\infty$ splits the elements $x \in K^{\star}$ into two cosets depending upon the sign of the embedding $x_{\infty}$. We let $\chi_{\infty}(x)=\operatorname{sign}\left(x_{\infty}\right)$ for elements $x \in K^{\star}$ and places $\infty \in \Omega_{K}^{\mathbf{R}}$, and note that these $\chi_{\infty}$ are multiplicative functions. By abuse of notation, for an ideal $\mathcal{I}$ and set $\Omega \subseteq \Omega_{K}^{\mathbf{R}}$, we write $\left(\mathbf{Z}_{K} / \mathcal{I} \Omega\right)^{\star}$ for the multiplicative group of invertible residue classes. We thus have

$$
\left(\mathbf{Z}_{K} / \mathcal{I} \Omega\right)^{\star} \cong \prod_{\mathfrak{p}^{k} \| \mathcal{I}}\left(\mathbf{Z}_{K} / \mathfrak{p}^{k}\right)^{\star} \times \prod_{\infty \in \Omega}\left(\mathbf{Z}_{K} / \infty\right)^{\star},
$$

and each of the terms in the latter product is isomorphic to $\mathbf{Z} / 2$.

2.1. Example. - We consider Dirichlet characters modulo $\mathcal{I}=(5)$ for the rational field $K=\mathbf{Q}$. There are four of these, and since 2 is a primitive root $\bmod 5$, we can specify such a character via its value at 2 , that is, $\chi(2)=\zeta_{4}^{i}$ for some $i$. Two of these characters are even (having $\chi(-1)=+1$ ), and two of them are odd (with $\chi(-1)=-1$ ).

We can then consider the four additional characters that appear when we take $\Omega=\{\infty\}$ to consist of the infinite place. We now have a character $\chi_{\infty}(x)=\operatorname{sign}(x)$, and its product with each of the previous four characters yields four new ones. In particular, we obtain four characters that are even, and thus trivial on the units, and two of these have $\infty$ in their conductor, and two do not. Since these are trivial on the units, they will induce characters on ideals when we consider Hecke characters. 
2.2. Induction and restriction of characters. - In general, we can specify a Dirichlet character modulo $\mathcal{I} \Omega$ by giving its image on generators for $\left(\mathbf{Z}_{K} / \mathcal{I} \Omega\right)^{\star}$. Thus it is easy to extend (or induce) a character $\chi$ to a larger modulus, as we simply note its values on generators for the new modulus.

The operation of restriction is not quite so easy, but also has more importance as it allows us to compute the conductor of a Dirichlet character. Given a Dirichlet character $\chi$ of modulus $\mathcal{I} \Omega$, we first decompose it into characters modulo prime powers $\mathfrak{p}^{k} \| \mathcal{I}$ and places in $\Omega$. We restrict to a modulus $\mathfrak{p}^{k}$ by computing generators for $\left(\mathbf{Z}_{K} / \mathfrak{p}^{k}\right)^{\star}$ and requiring that $\chi_{\mathfrak{p}} \equiv \chi$ on these, while $\chi_{\mathfrak{p}} \equiv 1$ on generators for $\left(\mathbf{Z}_{K} /\left(\mathcal{I} \Omega / \mathfrak{p}^{k}\right)\right)^{\star}$, and together these give us the desired behavior on $I \Omega$ via the Chinese remainder theorem. For infinite places $\infty \in \Omega$, it suffices simply to take $\chi_{\infty}$ as above, though it is also convenient to determine generators for $\left(\mathbf{Z}_{K} / \mathcal{I} \Omega_{\widehat{\infty}}\right)^{\star}$ where $\Omega_{\widehat{\infty}}=\Omega \backslash\{\infty\}$.

Given a Dirichlet character $\chi$ of prime power modulus $\mathfrak{p}^{k}$, we then want to compute the smallest power $\mathfrak{p}^{l}$ for which it is a character (that is, trivial on $1+\mathfrak{p}^{l} \mathbf{Z}_{K}$ ). We can assume that $k \geq 2$ and $l \geq 1$, as else the problem is not difficult. We then iteratively let $l$ decrease from $k$ to 1 , and at each step write down generators for the quotient of multiplicative groups $\frac{\left(1+\mathfrak{p}^{l-1} \mathbf{Z}_{K}\right)}{\left(1+\mathfrak{p}^{l} \mathbf{Z}_{K}\right)}$ and determine if $\chi$ is trivial on them. This quotient is isomorphic to the additive group of $\mathbf{F}_{\mathfrak{p}} \cong \mathbf{F}_{p}^{f}$ where $\mathrm{Np}=p^{f}$, and this simplifies the calculation. Upon finding the largest $l$ for which $\chi$ is nontrivial on this quotient, we conclude that the local conductor is $\mathfrak{p}^{l}$.

2.2.1. Use of Dirichlet characters. - The value of Dirichlet characters is somewhat limited as they do not have $L$-functions attached to them unless they are trivial on the units. One application of them would be to Hilbert modular forms with character.

\section{Hecke characters}

We now pass to Hecke characters, which are characters on ideals. In particular, a Dirichlet character only lifts to a Hecke character if it is trivial on all the units of $K$. We must also consider characters corresponding to the class group $\mathcal{C} l_{K}$ of $K$.

The ray class group modulo $\mathcal{I}$ is defined as the quotient of the ideals of $\mathbf{Z}_{K}$ that are coprime to $\mathcal{I}$ by the principal ideals $(\alpha)$ for $\alpha \equiv 1(\bmod \mathcal{I})$. We can further include information about ramification at infinite places by requiring that such an $\alpha$ be positive at places in a set $\Omega$. Given the standard multiplication operation on ideals, the ray class group is thus

$$
R_{\mathcal{I} \Omega}=\frac{\left\{\mathfrak{a} \subseteq \mathbf{Z}_{K}, \operatorname{gcd}(\mathfrak{a}, \mathcal{I})=1\right\}}{\left\{(\alpha): \alpha \equiv 1(\bmod \mathcal{I}), \text { and } \alpha_{\infty}>0 \text { for all } \infty \in \Omega\right\}},
$$

and we have the following diagram:

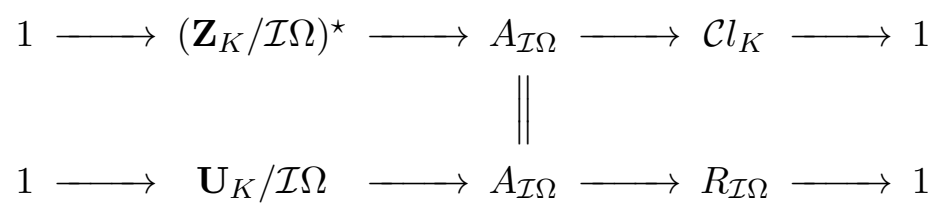

where $A_{\mathcal{I} \Omega}$ is a group extension of $\mathcal{C} l_{K}$ by $\left(\mathbf{Z}_{K} / \mathcal{I} \Omega\right)^{\star}$ that we describe more fully below, and $\mathbf{U}_{K}$ is the unit group of $K$. 
The Hecke character group modulo $\mathcal{I} \Omega$ will be dual to $R_{\mathcal{I} \Omega}$ in the above, and as there is a natural quotient map from $R_{\mathcal{I} \Omega}$ onto $\mathcal{C l}_{K}$, there will be a natural injection from the Hilbert character group $\widehat{\mathcal{C} l_{K}}$ into the Hecke character group. Similarly, though not of as much import, there is an injection from $\mathbf{U}_{K}$ into $\mathbf{Z}_{K}$ that yields a quotient map from the Dirichlet character group modulo $\mathcal{I} \Omega$ onto the unit characters $\widehat{\mathbf{U}_{K} / \mathcal{I}} \Omega$.

3.1. The group extension $A_{\mathcal{I} \Omega}$. - We now say a bit more about the group extension $A_{\mathcal{I} \Omega}$. To construct this, we take representatives $\mathfrak{a}_{i}$ that generate the class group $\mathcal{C} l_{K}$ and are coprime to $\mathcal{I}$. For each $i$ we let $o_{i}$ be the order of $\mathfrak{a}_{i}$ in $\mathcal{C} l_{K}$, and we principalise $\mathfrak{a}_{i}^{o_{i}}=\left(u_{i}\right)$ with $\left(u_{i}\right)_{\infty}>0$ for all $\infty \in \Omega$ and take $\mathfrak{a}_{i}^{o_{i}}=u_{i}$ as a relation in $A_{\mathcal{I} \Omega}$. We see that $u_{i}$ is defined only up to units in general, but any ambiguity disappears when we take the quotient by $\mathbf{U}_{K}$. However, we still do have some choices with the various class group representatives. The computation of ray class groups is detailed in [12], and eventually reduces to computing discrete logarithms.

3.2. Dirichlet restriction and Hecke lifting. - We can restrict a Hecke character $\psi$ to a Dirichlet character $\chi$ of the same modulus $\mathcal{I} \Omega$ in an obvious way, simply by putting $\chi=\psi$ on a set of generators for $\left(\mathbf{Z}_{K} / \mathcal{I} \Omega\right)^{\star}$. The reverse process lifts a Dirichlet character that is trivial on all units that are positive at all places in $\Omega$, and yields a Hecke character that is well-defined up to a Hilbert character.

3.3. Hecke character restriction and extension. - As with the Dirichlet character case, we often find it convenient to define a Hecke character $\psi$ of modulus $\mathcal{I} \Omega$ via giving values on generators for $R_{\mathcal{I} \Omega}$. This immediately gives us a method to restrict a Hecke character to a smaller modulus. When extending a Hecke character, the calculation of relative generators for the Chinese remainder theorem can be a bit delicate, and it seems easier to work via extending its Dirichlet restriction to the larger modulus as before and then combine this with evaluations of $\psi$ on generators of $R_{\mathcal{I} \Omega}$ (see $\S 3.5$ ).

3.3.1. Lack of Hecke decompositions. - The conductor of a Hecke character is simply the conductor of its Dirichlet restriction, as the only information lost is that of a Hilbert character, whose conductor is trivial. There is really no precise idea of a "decomposition" for Hecke characters, due to the possible interaction of units when restricting the modulus. An easy example of this phenomenon is to take $K=\mathbf{Q}(\sqrt{37})$ and $\mathcal{I}=(3)$ and $\mathcal{J}=(5)$. Then $R_{\mathcal{I}}$ has order 1 and $R_{\mathcal{J}}$ has order 2 , while $R_{\mathcal{I} \mathcal{J}}$ is cyclic of order 4 . Here we have that both $R_{\mathcal{J}}$ and $R_{\mathcal{I J}}$ are generated by $(\sqrt{37})$. We see that the units $\{-1,6+\sqrt{37}\}$ generate the multiplicative group $\left(\mathbf{Z}_{K} / \mathcal{I}\right)^{\star}$, while they form subgroups of indices 2 and 4 respectively when considered similarly modulo $\mathcal{J}$ and $\mathcal{I} \mathcal{J}$.

3.4. Example. - Of the eight Dirichlet characters of $\mathbf{Q}$ modulo (5) $\infty$ given in the previous example, four of these are trivial on the units, and these lift to Hecke characters. Two of them have $\infty$ in their conductor.

3.5. Explicit lifting. - Take $K=\mathbf{Q}(\sqrt{229})$ which has class number 3 and $\mathcal{I}=(7)$. Then we have that $\widehat{R_{\mathcal{I}}} \cong \mathbf{Z} / 3 \times \mathbf{Z} / 3$, and we can identify one of these constituents with the Hilbert characters. We can define $\psi$ by $\psi\left(\mathfrak{p}_{3}\right)=\psi(2)=\zeta_{3}$, though this still leaves ambiguity 
regarding $\mathfrak{p}_{3}$ versus its conjugate, and similarly with $\zeta_{3}$. The Dirichlet group modulo $\mathcal{I}$ is cyclic of order 48 , and $\psi$ restricts to an element $\chi$ of order 3 , which can be defined by $\chi(2)=\zeta_{3}$ and $\chi(2+\sqrt{229})=1$.

We then extend $\chi$ to modulus $\mathcal{I} \mathcal{J}$ where $\mathcal{J}=\mathfrak{p}_{19}$ is a prime above 19 . The Dirichlet group here is isomorphic to $\mathbf{Z} / 48 \times \mathbf{Z} / 18$, where the element 2 has order 18 while $2+\sqrt{229}$ has order 144, and these generate (this is dependent on choosing $\mathcal{J}$ rather than its conjugate). So we can define the lift of $\psi$ to $\mathcal{I} \mathcal{J}$ by $\psi(2)=\psi\left(\mathfrak{p}_{3}\right)=\zeta_{3}$ and $\psi(2+\sqrt{229})=1$.

3.6. L-function and functional equation. - Let $\psi$ be a Hecke character of conductor $\mathfrak{c} \Omega$; we take $\psi$ to be primitive so that the conductor and modulus are equal. The $L$-function of $\psi$ is given as an Euler product and Dirichlet series (convergent in some half-plane) as

$$
L(\psi, s)=\prod_{\mathfrak{p}}\left(1-\psi(\mathfrak{p}) / \mathrm{Np}^{s}\right)^{-1}=\sum_{\mathfrak{a} \subseteq \mathbf{Z}_{K}} \frac{\psi(\mathfrak{a})}{\mathrm{Na}^{s}},
$$

conventionally taking $\psi(\mathfrak{a})=0$ for ideals $\mathfrak{a}$ that are not coprime to $\mathfrak{c}$.

We define $\Gamma_{\mathbf{R}}(s)=\frac{\Gamma(s / 2)}{\pi^{s / 2}}$ and $\Gamma_{\mathbf{C}}(s)=\Gamma_{\mathbf{R}}(s) \Gamma_{\mathbf{R}}(s+1)=2 \frac{\Gamma(s)}{(2 \pi)^{s}}$, and write

$$
L_{\infty}(\psi, s)=\prod_{\infty \in \Omega_{K}^{\mathbf{C}}} \Gamma_{\mathbf{C}}(s) \cdot \prod_{\substack{\infty \in \Omega_{K}^{\mathbf{R}} \\ \infty \notin \Omega}} \Gamma_{\mathbf{R}}(s) \cdot \prod_{\substack{\infty \in \Omega_{K}^{\mathbf{R}} \\ \infty \in \Omega}} \Gamma_{\mathbf{R}}(s+1) .
$$

We then have that the completed $L$-function has a meromorphic continuation and satisfies

$$
\begin{gathered}
\Lambda(\psi, s)=\epsilon_{\psi} \Lambda(\bar{\psi}, 1-s) \quad \text { for some } \epsilon_{\psi} \text { with }\left|\epsilon_{\psi}\right|=1 \text {, where } \\
\Lambda(\psi, s)=L(\psi, s) \cdot\left(\mathrm{Nc} \cdot\left|\Delta_{K}\right|\right)^{s / 2} \cdot L_{\infty}(\psi, s) .
\end{gathered}
$$

We can also note we obtain the Dedekind $\zeta$-function for $K$ here by taking $\psi$ to be the trivial Hecke character - this is the only case where the $L$-function has a pole.

3.6.1. Root numbers. - We can quote the formula for root numbers from Tate's thesis [23]. The global root number $\epsilon_{\psi}$ is a product $\prod_{v} \epsilon_{v}(\psi)$ of local root numbers, where $\epsilon_{v}(\psi)=+1$ when $v$ is finite and neither $\psi$ nor $K$ is ramified at it. We have three cases: $v$ is archimedean, when $\epsilon_{v}(\psi)=e^{2 \pi i / 4}$ when $v$ is real archimedean and $v \in \Omega$, and else $\epsilon_{v}(\psi)=+1$ (see [5, $\S 5.3]) ; \psi$ is unramified at $v$ finite, when $\epsilon_{v}(\psi)=\psi\left(\mathfrak{d}_{v}\right)^{-1}$ where $\mathfrak{d}_{v}$ is the local different of $K_{v}$; and $\psi$ is ramified at $v$ finite, when we get a Gauss sum for the local character $\psi_{v}$ :

$$
\epsilon_{v}(\psi)=\frac{1}{\sqrt{\mathrm{Nc}_{v}}} \sum_{a} \psi_{v}(a) e^{2 \pi i \cdot \operatorname{tr}\left(a / \pi_{v}^{e}\right)},
$$

where $a$ runs over $\mathbf{Z}_{v} / \mathfrak{c}_{v}$ (or its multiplicative group), while $\pi_{v}$ is a uniformising element and $\pi_{v}^{e}$ is a generator for the ideal $\mathfrak{d}_{v} \mathfrak{c}_{v}$, and finally the trace includes the canonical maps $\mathbf{Q}_{p} \rightarrow \mathbf{Q}_{p} / \mathbf{Z}_{p} \hookrightarrow \mathbf{Q} / \mathbf{Z} \hookrightarrow \mathbf{R} / \mathbf{Z}$. Note that $\epsilon_{\psi}$ is algebraic, though it is given by a specific embedding (given by the explicit $e^{2 \pi i}$ in the above, rather than an arbitrary root of unity). In the case of Hilbert characters, we note that the above reduces to $\epsilon_{\psi}=\psi\left(\mathfrak{d}_{K}\right)^{-1}$, which leads to various characterisations. For instance, when we have an integral power-basis $\mathbf{Z}_{K}=\mathbf{Z}[\alpha]$, then the global different $\mathfrak{d}_{K}$ is principal (letting $f$ be a minimal polynomial for $\alpha$, we have that $\mathfrak{d}_{K}=\left(f^{\prime}(\alpha)\right)$ in this case), and so $\epsilon_{\psi}=+1$ for all Hilbert characters. The fact that the global different is always a square in the class group (see [11, Satz 176]) can also be exploited 
in some cases. An example of a Hilbert character with nontrivial root number is with the field $K=\mathbf{Q}(\sqrt[3]{175})$, where the class group has order 3 and the root numbers of the Hilbert characters are the cube roots of unity. The different here is $\mathfrak{d}_{K}=\mathfrak{p}_{3}^{3} \mathfrak{p}_{5}^{2} \mathfrak{p}_{7}^{2}$, with $\mathfrak{p}_{5}$ and $\mathfrak{p}_{7}$ in the same ideal class since $\mathfrak{p}_{5}^{2} \mathfrak{p}_{7}=(\sqrt[3]{175})$ is principal.

3.6.2. Critical values. - A critical point for a Hecke $L$-function is an integer $n$ such that neither $L_{\infty}(s)$ nor $L_{\infty}(1-s)$ has a pole at $s=n$. The $L$-function of a (primitive) Hecke character $\psi$ has critical points only when $K$ is totally real and either all or none of the infinite places are contained in $\Omega$. Writing $\mathbf{Q}(\psi)$ for the value-field of $\psi$, a theorem of Siegel [22] (see also $[4, \S 1.2])$ refines work of Klingen and states that $L(\psi,-2 k-\epsilon) \in \mathbf{Q}(\psi)$ for all nonnegative integers $k$, where $\epsilon=1$ when $\Omega$ is empty, and $\epsilon=0$ when $\Omega$ contains all the real places.

3.7. $L$-function example. - We take $K=\mathbf{Q}(\sqrt{-23})$ of class number 3, and consider either of the nontrivial Hilbert characters $\psi$. Thus any nonprincipal ideal gets mapped to $\zeta_{3}$ or $\zeta_{3}^{2}$. Using a technique dating back to Hecke made explicit by Lavrik [14] we can write $L(\psi, 1)$ as a rapidly converging series, and so approximate it as

$$
L(\psi, 1) \approx 0.368409320715826821111868466629 .
$$

Writing $S$ for the (non-Galois) cubic subfield of the Hilbert class field of $K$, we can note that

$$
\zeta_{S}(s)=\zeta_{\mathbf{Q}}(s) \cdot L(\psi, s),
$$

and this allows us to use the residue formula for the Dedekind $\zeta$-function to get that $L(\psi, 1)=$ $\frac{2 \pi \log \epsilon}{\sqrt{23}}$ where $\epsilon$ is the real root of $x^{3}-x-1$. We could alternatively write $L(\psi, s)$ as the $L$ function of a 2-dimensional Artin representation, but do not pursue such avenues herein.

3.8. An example with root numbers and critical values. - Let $K=\mathbf{Q}(\sqrt{13})$ and take $\psi$ to be either nontrivial Hecke character modulo $\mathfrak{p}_{13}$. At the bad prime $\mathfrak{p}_{13}$ we have that $\mathfrak{d}_{\mathfrak{p}_{13}} \mathfrak{c}_{\mathfrak{p}_{13}}=(13)=(\sqrt{13})^{2}$, and so the root number $\epsilon_{\psi}=\epsilon_{\mathfrak{p}_{13}}$ is just given by $\frac{1}{\sqrt{13}} \sum_{j} \psi(j) e^{2 \pi i(2 j / 13)}$, where the sum is over residues $\bmod 13$. This is a root of $x^{12}+$ $\frac{1}{13} x^{6}+1$, and an approximation is

$$
0.711626069061866188779523 \pm 0.7025584230735235114677424 i .
$$

We compute $L(\psi,-1)=\frac{4}{13}\left(7-11 \zeta_{3}\right)$ and $L(\psi,-3)=\frac{4}{13}\left(3883-9491 \zeta_{3}\right)$.

Upon taking $\psi$ to be either Hecke character with conductor $\mathfrak{p}_{13} \infty_{1} \infty_{2}$, the global root number is approximately

$$
-0.8723658594356627130720628 \pm 0.4888535642614028900637261 i,
$$

and at $\mathfrak{p}_{13}$ we now get $\epsilon_{\mathfrak{p}_{13}}$ as a root of $x^{6}-\frac{1}{13} x^{3}+1$, and we also have a contribution of $i^{2}=-1$ from the two real infinite places. The first two critical values can be computed to be $L(\psi, 0)=\frac{4}{13}\left(1+4 \zeta_{3}\right)$ and $L(\psi,-2)=12\left(-4+5 \zeta_{3}\right)$.

3.8.1. Relation of root numbers to other Dirichlet characters. - The reader can note that we get similar root numbers for the classical Dirichlet characters modulo 13. Indeed, we have two such characters of order 3 , but the above root number formula would have $e^{2 \pi i(j / 13)}$, while in our case we have $2 j$ in the exponent. 
3.9. PARI/GP implementation of Hecke characters. - There is a package of Maciej Radziejewski that implements Hecke characters in PARI/GP. See [15] for more details.

\section{Hecke Grössencharacters}

We largely follow Chapter Zero of [19], and we will only deal with algebraic Grössencharacters, or those of type $A_{0}$. We let $K$ be a number field, which we will eventually take to be a CMfield, that is, a totally imaginary quadratic extension of a totally real field.

An $\infty$-type $\mathbf{T}$ is a sequence of integers $\left(n_{\sigma}\right)$ indexed by the embeddings $\sigma$ of $K$. The evaluation of an $\infty$-type at an element $\alpha \in K$ is given by $\mathbf{T}(\alpha)=\prod_{\sigma}\left(\alpha^{\sigma}\right)^{n_{\sigma}}$. Note that the norm is the $\infty$-type with all components equal to 1 , and so we can easily renormalise to have (say) all the components nonnegative and at least one of them equal to zero. We require that $n_{\sigma}+n_{\bar{\sigma}}=w$ be constant over all embeddings $\sigma$, where here $w$ is called the weight.

For a given modulus $\mathcal{I} \Omega$, we consider the set of "coherent" $\infty$-types modulo $\mathcal{I} \Omega$ on $K$ that is, the $\infty$-types for which $\mathbf{T}(\alpha)=1$ for all units $\alpha \equiv 1(\bmod \mathcal{I})$ with $\alpha_{\infty}>0$ for all $\infty \in \Omega$. Every algebraic Hecke character factors through the relative norm from $K$ to the maximal CM-subfield of $K$ (see $[\mathbf{1 9}, \S 0.3]$ ), and so unless $K$ contains a CM-field, the only coherent infinity types will be multiples of the norm. For simplicity we will assume that $K$ is itself CM from now on, and so $\Omega$ is empty (as there are no real places). We can also pair the embeddings in the $\infty$-type according to complex conjugacy.

Given a modulus $\mathcal{I}$ and a coherent $\infty$-type $\mathbf{T}$, we then define

$$
\mathbf{G}((\alpha))=\mathbf{T}(\alpha)=\prod_{\sigma}\left(\alpha^{\sigma}\right)^{n_{\sigma}}
$$

for all $\alpha \equiv 1(\bmod \mathcal{I})$. This defines a Hecke Grössencharacter $\mathbf{G}$ up to a finite quotient that is exactly the Hecke character group for $\mathcal{I}$. Indeed, what we called Hecke characters are often called "finite order Hecke characters" for this reason. We again conventionally take $\mathbf{G}(\mathfrak{a})=0$ on the ideals $\mathfrak{a}$ that are not coprime to $\mathcal{I}$. We can also note that $\mathbf{G}(\mathfrak{p})$ will be of size $(\mathrm{Np})^{w / 2}$ for $\mathfrak{p}$ that are coprime to $\mathcal{I}$.

In general, the values of $\mathbf{G}$ will lie in an extension $E / K$ given by roots of principalisations of powers of representatives of the class group. Furthermore, twisting by a (finite order) Hecke character mod $\mathcal{I}$ can enlarge the value-field by an additional cyclotomic factor.

4.1. Example. - The typical first examples here are for imaginary quadratic fields of class number one, and are related to elliptic curves with complex multiplication. For instance, upon taking $K=\mathbf{Q}(\sqrt{-1})$ and $\mathcal{I}=\mathfrak{p}_{2}^{3}$ where $\mathfrak{p}_{2}$ is the ramified prime above 2 , with $T=[1,0]$ for the $\infty$-type we get a Hecke Grössencharacter whose $L$-series matches that of the congruent number curve. The condition $\alpha \equiv 1(\bmod \mathcal{I})$ can be described in terms of primary generators in this case.

In this context, we can mention the "canonical" Grössencharacters of Rohrlich [17] for imaginary quadratic fields, which are closely related to Hilbert characters. 


\section{Implementation of Grössencharacters}

We next turn to how compute with Grössencharacters, and this will actually help explain some technical theoretical points. The implementation of Dirichlet and Hecke characters needs nothing more than to be able to compute generators for the residue and ray class groups, and then to be able to compute discrete logs with them. For Grössencharacters we also need to be able to principalise ideals. We also have a choice of how the extension field is embedded into $\mathbf{C}$, though such embeddings are permuted by Hilbert characters (see below).

5.1. First method for Grössencharacters. - The most direct method to compute Grössencharacters modulo $\mathcal{I}$ for a coherent $\infty$-type $\mathbf{T}$ of a field $K$ is to write down representatives $\mathfrak{a}_{i}$ of a basis for the ray class group $R_{\mathcal{I}}$, and extend $K$ by the roots $\mathbf{T}\left(u_{i}\right)^{1 / o_{i}}$ where $o_{i}$ is the order of $\mathfrak{a}_{i}$ in $R_{\mathcal{I}}$ and $\left(u_{i}\right)=\mathfrak{a}_{i}^{o_{i}}$ is a principalisation with $u_{i} \equiv 1(\bmod \mathcal{I})$. Here by $\mathbf{T}\left(u_{i}\right)^{1 / o_{i}}$ we mean a specific choice of root in an extension field.

We concentrate on the principal Grössencharacter $\mathbf{G}$, as the others can be determined via the finite quotient corresponding to $R_{\mathcal{I}}$. We take $\mathbf{G}\left(\mathfrak{a}_{i}\right)=\mathbf{T}\left(u_{i}\right)^{1 / o_{i}}$ and can compute $\mathbf{G}$ on any ideal $\mathfrak{b}$ with $\operatorname{gcd}(\mathfrak{b}, \mathcal{I})=1$ by using the rule

$$
\mathbf{G}(\mathfrak{b})=\mathbf{G}\left(\mathfrak{b} \cdot \prod_{i} \mathfrak{a}_{i}^{v_{i}}\right) / \mathbf{G}\left(\prod_{i} \mathfrak{a}_{i}^{v_{i}}\right) .
$$

Here the $b_{i}$ are chosen to make $\mathfrak{b} \prod_{i} \mathfrak{a}_{i}^{v_{i}}$ lie in the trivial class of $R_{\mathcal{I}}$, with the value of $\mathbf{G}$ on such classes then determined (after principalisation) by the $\infty$-type as in (2).

We can note that the choices of $\mathfrak{a}_{i}$ do not make too much difference. Indeed, if $\mathfrak{a}$ and $\mathfrak{b}$ are in the same class with $\mathfrak{a}^{o}=\left(u_{\mathfrak{a}}\right)$ and $\mathfrak{b}^{o}=\left(u_{\mathfrak{b}}\right)$, then $K\left(\mathbf{T}\left(u_{\mathfrak{a}}\right)^{1 / o}\right)$ and $K\left(\mathbf{T}\left(u_{\mathfrak{b}}\right)^{1 / o}\right)$ are isomorphic fields. This follows because we can write $\mathfrak{a} / \mathfrak{b}=(t)$ with $t \equiv 1(\bmod \mathcal{I})$, so that $t^{o} \xi=u_{\mathfrak{a}} / u_{\mathfrak{b}}$ with $\xi \equiv 1(\bmod \mathcal{I})$ a unit, which yields $\mathbf{T}\left(u_{\mathfrak{b}}\right)=t^{o} \mathbf{T}(\xi) \mathbf{T}\left(u_{\mathfrak{a}}\right)$ where $\mathbf{T}(\xi)=$ 1 , implying the result.

However, there are two problems with this method. The first is that the extension field can be of quite large degree, and the second is that it is difficult to pass from one modulus to another.

5.2. Second method for Grössencharacters. - A superior method is to realise the above "translation" (3) in the class group of $K$ rather than in the ray class group, though we then need to take care with units.

Given a CM-field $K$, we choose representatives $\mathfrak{a}_{i}$ of a basis of the class group. We write $o_{i}$ for the orders, and $\left(u_{i}\right)=\mathfrak{a}_{i}^{o_{i}}$ for principalisations. Given an $\infty$-type $\mathbf{T}$ we then let $\chi_{\mathbf{U}_{K}}^{\mathbf{T}}$ be a character on the units $\mathbf{U}_{K}$ such that $\chi_{\mathbf{U}_{K}}^{\mathbf{T}}(\epsilon)=\mathbf{T}(\epsilon)^{-1}$ for all units $\epsilon \in K$. We define the value-field $E$ of $K(\mathbf{T})$ to be the compositum of the $K_{i}=K\left(\mathbf{T}\left(u_{i}\right)^{1 / o_{i}}\right)$, where we make specific choices of roots. The value-field of a Grössencharacter G can firstly "twist" $K(\mathbf{T})$ to use different choices of roots, and additionally can include a cyclotomic extension corresponding to a nontrivial choice of a finite order Hecke character.

We now introduce the modulus $\mathcal{I}$ and let $\chi$ be a Dirichlet character modulo $\mathcal{I}$ that is a lift of $\chi_{\mathbf{U}_{K}}^{\mathbf{T}}$. We may try to take $\chi$ to be "quasi-minimal" in the sense that we define it as above for generators of $\mathbf{U}_{K} / \mathcal{I}$, and trivial on other factors in the $\left(\mathbf{Z}_{K} / \mathcal{I}\right)^{\star}$ decomposition. All possible lifts will differ by a finite order Hecke character, as the quotient of any two lifts will 
be trivial on the units. We can still have multiple "quasi-minimal" choices for $\chi$ - one case is $K=\mathbf{Q}(\sqrt{-15})$ with $\mathcal{I}=\mathfrak{p}_{3} \mathfrak{p}_{5}$, where either quadratic character modulo $\mathcal{I}$ with $\chi(-1)=-1$ suffices.

It is convenient to choose an lift $\tilde{\chi}$ of $\chi$ to $\widehat{A}_{\mathcal{I}}$ (recall diagram $\left.(1)\right)$, and we choose $\tilde{\chi}\left(\mathfrak{a}_{i}, u_{i}\right)=$ $\chi\left(u_{i}\right)^{1 / o_{i}}$ for some choice of root of unity on the right. Since the group extension is by the class group, it follows that Hilbert characters will permute these choices of roots.

Given a character-lift $\tilde{\chi}$, the resulting "principal" Grössencharacter $\mathbf{G}$ modulo $\mathcal{I}$ will give $\mathbf{G}\left(\mathfrak{a}_{i}\right)=\mathbf{T}\left(u_{i}\right)^{1 / o_{i}} \tilde{\chi}\left(\mathfrak{a}_{i}, u_{i}\right)$ when evaluated at the class group representatives $\mathfrak{a}_{i}$, while the value at a principal ideal $(v)$ will be $\mathbf{G}((v))=\mathbf{T}(v) \chi(v)$. We can compute the value at any other ideal as with the above translation (3). We have $(v)=(\epsilon v)$ for any unit $\epsilon \in K$, and can check that we do indeed have

$$
\mathbf{G}((\epsilon v))=\mathbf{T}(\epsilon v) \chi(\epsilon v)=\mathbf{T}(\epsilon) \mathbf{T}(v) \chi(\epsilon) \chi(v)=\mathbf{T}(v) \chi(v)=\mathbf{G}((v)) .
$$

We will also want our representatives $\mathfrak{a}_{i}$ to be coprime to a given ideal $\mathcal{I}$, and will want to be able to change our chosen representatives in some cases. As an example, we take $K=$ $\mathbf{Q}(\sqrt{-23})$ and suppose we choose $\mathfrak{p}_{2}$ as a class group representative for a calculation with some $\mathbf{G}_{3}$ modulo (3), and $\mathfrak{p}_{3}$ for a calculation with some $\mathbf{G}_{2}$ modulo (2). To work with $\mathbf{G}_{3} \mathbf{G}_{2}$ we want to convert both $\mathbf{G}_{2}$ and $\mathbf{G}_{3}$ to use a representative that is coprime to 6 , say $\mathfrak{p}_{13}$. We achieve this simply via $\mathbf{G}_{3}\left(\mathfrak{p}_{13}\right)=\mathbf{G}_{3}\left(\mathfrak{p}_{13} / \mathfrak{p}_{2}\right) \mathbf{G}_{3}\left(\mathfrak{p}_{2}\right)$, and similarly with 2 and 3 switched. We can then compute either $\mathbf{G}_{2}$ or $\mathbf{G}_{3}$ with $\mathfrak{p}_{13}$ rather than the original choices of class group representatives.

5.3. L-function, functional equation. - Attached to a Grössencharacter $\mathbf{G}$ we thus have an $\infty$-type $\mathbf{T}$, a modulus $\mathcal{I}$, a finite order Hecke character $\psi$, and a Dirichlet character $\chi$, though these last two can be combined into the lift $\tilde{\chi}$ if desired. The conductor of such a Grössencharacter is the conductor of the product of $\chi$ with the Dirichlet restriction of $\psi$. The value-field $K(\mathbf{G})$ is given by $K(\mathbf{T} \chi, \psi)$, where here $K(\mathbf{T} \chi)$ is the field generated by the $\mathbf{G}\left(\mathfrak{a}_{i}\right)$. The $L$-function of a Grössencharacter is defined as $\prod_{\mathfrak{p}}\left(1-\mathbf{G}(\mathfrak{p}) / \mathrm{Np}^{s}\right)^{-1}$, and we note that $L(\mathbf{G}, s)=L(\overline{\mathbf{G}}, s)$ when $\mathbf{G}(\overline{\mathfrak{p}})=\overline{\mathbf{G}}(\mathfrak{p})$ for all primes $\mathfrak{p}$, as conjugate pairs appear together in the Euler product. The functional equation is of a similar form to that for finite order Hecke characters, though we now have the completed $L$-function satisfies $\Lambda(\mathbf{G}, s)=\epsilon_{\mathbf{G}}$. $\Lambda(\overline{\mathbf{G}}, w+1-s)$. For the $\Gamma$-factor, we take each conjugate pair $[p, q]$ in the $\infty$-type and write it with $p \leq q$ (this is permissible via relabelling the embeddings in the conjugate pair), and each then gives a factor of $\Gamma_{\mathbf{C}}(s-p)$. Note that $\overline{\mathbf{G}}$ has the conjugate $\infty$-type to that of $\mathbf{G}$, so that it has the same $\Gamma$-factors. Assuming that $\mathbf{G}$ has been scaled to remove factors of the norm, the completed $\Lambda$-function has a pole precisely when $\mathbf{G}$ is the trivial Grössencharacter of weight 0

5.3.1. Root numbers. - The root number formulæ of above also need to be modified slightly. At (complex) infinite places $v$ of type $[p, q]$ (again taking $p \leq q$ ) we have $\epsilon_{v}(\mathbf{G})=i^{q-p}$ $[\mathbf{5}, \S 5.3]$, while at finite places $v$ where $K$ is ramified but $\mathbf{G}$ is not the local root number is $\epsilon_{v}(\mathbf{G})=\mathbf{G}\left(\mathfrak{d}_{v}\right) / \mathrm{Nd}_{v}=\tilde{\mathbf{G}}\left(\mathfrak{d}_{v}\right)$. Finally, at finite places $v$ where $\mathbf{G}$ is ramified the previous Gauss sum now has $\psi_{v}$ replaced by $(\psi \chi)_{v}$. There are various useful formulæ relating twists of $L$-series, of which we mention that if $\psi$ is finite and $\operatorname{gcd}\left(\mathfrak{c}_{\psi}, \mathfrak{c}_{\mathbf{G}}\right)=1$, then

$$
\epsilon_{G \psi}=\epsilon_{G} \cdot \epsilon_{\psi} \cdot \psi^{-1}\left(\mathfrak{c}_{\mathbf{G}}\right) \cdot \tilde{\mathbf{G}}^{-1}\left(\mathfrak{c}_{\psi}\right)
$$




\subsubsection{Critical values. -}

The critical points (for the $L$-functions) of these Hecke Grössencharacters are those integers $n$ such that neither $L_{\infty}(s)$ nor $L_{\infty}(w+1-s)$ has a pole at $s=n$. It follows that the critical points for a Grössencharacter of $\infty$-type $[0, w]$ are $s=1 \ldots w$, and in general they are $s=$ $(\mu+1) \ldots(w-\mu)$ where $\mu$ is the largest $p$ amongst the $[p, q]$ conjugate pairs (again taking $p \leq q$ in each). For the right half of the critical points, that is, the critical $u$ with $u \geq(w+1) / 2$ (for else the root number intervenes), a conjecture of Deligne $[\mathbf{5}, \S 2.8, \S 8]$ states that there is a period $\omega(\mathbf{G}) \in \mathbf{C}$ such that $L(\mathbf{G}, u)(2 \pi i)^{w-u} / \omega(\mathbf{G})$ is in the value-field of $\mathbf{G}$, and this is a theorem of Blasius [1] (refining work of Shimura [21], while Goldstein and Schappacher [7] handled the case where $K$ is imaginary quadratic).

A more complete statement here can be made by first defining $\omega_{K}^{\mathbf{T}}$ for a CM-field $K$ and $\infty$-type $\mathbf{T}$ via periods of an abelian variety (of dimension $\frac{1}{2}[K: \mathbf{Q}]$ ) that has CM by $K$ and is defined over the Hilbert class field $H / K$. This is not completely straightforward, as the periods will naturally lie in $H \otimes \mathbf{C}$, and we want them in $K(\mathbf{G}) \otimes \mathbf{C}$. After obtaining the period $\omega_{K}^{T}$, we can then relate it to $\omega(\mathbf{G})$ for any $\mathbf{G}$ with $\infty$-type $\mathbf{T}$. We go through this process in some detail in Example 6.1 below, first following $[\mathbf{7}, \S 4]$ to get an explicit $\omega_{K}^{[2,0]}$ in $K=\mathbf{Q}(\sqrt{-23})$, and then relating it to a Grössencharacter with this $\infty$-type and trivial conductor.

In fact, since we have $\omega_{K}^{\mathbf{T}_{1}} \omega_{K}^{\mathbf{T}_{2}}=\omega_{K}^{\mathbf{T}_{1}+\mathbf{T}_{2}}$, all the $\omega_{K}^{\mathbf{T}}$ will be generated by those on a basis for the $\infty$-types, namely all $\infty$-types of weight 1 (that is, all conjugate pairs as $[0,1]$ or $[1,0])$. This basis has size $[K: \mathbf{Q}]$, and can be halved upon considering complex conjugation, in exact correspondence with the periods from the abelian variety. The multiplication formula $\omega\left(\mathbf{G}_{1} \mathbf{G}_{2}\right)=\omega\left(\mathbf{G}_{1}\right) \omega\left(\mathbf{G}_{2}\right)$ is true up to a determinable factor in $K(\mathbf{G})$ (see $[\mathbf{1 9}$, II.1.8.1-3], with multiplicativity of the $p$, and their relation to the periods), while the effect of twisting by a finite order Hecke character can also be determined and adds at worst an abelian extension (see [19, II.3], and note that the last sentence in $[\mathbf{9}, \S 4]$ is incorrect, as per $[\mathbf{1 9}$, II.3.4]).

The net result is that $L(\mathbf{G}, u)(2 \pi i)^{w-u} / \omega(\mathbf{G}) \in K(\mathbf{G})$, where both $L(\mathbf{G}, u)$ and $\omega(\mathbf{G})$ are elements of $K(\mathbf{G}) \otimes \mathbf{C}$ that can be independently computed.

5.4. An example. - Let $K=\mathbf{Q}(\sqrt{-23})$ and $\mathcal{I}=\mathfrak{p}_{23}$ be the ramified prime. We consider the principal Grössencharacter $\mathbf{G}$ with $\infty$-type [1,0], taking the Dirichlet character $\chi$ to be the quadratic character modulo $\mathfrak{p}_{23}$. The lift $\tilde{\chi}$ can be taken to be the trivial extension of $\chi$, as the orders of the class group and unit group are coprime.

We take $\mathfrak{p}_{2}$ as our representative for the class group generator, and principalise $\mathfrak{p}_{2}^{3}=$ $\left(\frac{3+\sqrt{-23}}{2}\right)=(u)$ so that $E=K\left(u^{1 / 3}\right)$. Letting $\mathfrak{p}_{3}$ be in the same class as $\mathfrak{p}_{2}$ in the class group, we compute that $\mathbf{G}\left(\mathfrak{p}_{3}\right)$ is equal to

$$
\mathbf{G}\left(\mathfrak{p}_{3} / \mathfrak{p}_{2}^{2}\right) \mathbf{G}\left(\mathfrak{p}_{2}^{2}\right)=\mathbf{G}\left(\left(\frac{5-\sqrt{-23}}{8}\right)\right) u^{2 / 3}=-\left(\frac{5-\sqrt{-23}}{8}\right) u^{2 / 3},
$$

the last step since $\chi\left(\frac{5-\sqrt{-23}}{8}\right)=-1$.

If we had chosen a different lift, say $\tilde{\chi}(u)=\zeta_{3}$, then we would have $\mathbf{G}\left(\mathfrak{p}_{2}^{2}\right)=u^{2 / 3} \zeta_{3}^{2}$, which corresponds to a different embedding for $E$ as an extension of $K$. We can also note that $\mathfrak{p}_{2}$ is only defined up to complex conjugacy. 
5.5. The choice of embedding and Hilbert characters. - We next consider $K=$ $\mathbf{Q}(\sqrt{-39})$ which has cyclic class group of 4 , and let $\psi$ be a Hilbert character of order 4 with $\psi\left(\mathfrak{p}_{2}\right)=\zeta_{4}$. Let $\chi$ be either of the (imprimitive) quadratic Dirichlet characters with modulus $\mathfrak{p}_{3} \mathfrak{p}_{13}$ and $\chi(-1)=-1$, and $\mathbf{G}$ be the principal Grössencharacter with $\infty$-type $[1,0]$. We take $\mathfrak{p}_{2}$ as our representative of class group generator where $\mathfrak{p}_{2}^{4}=\left(\frac{5+\sqrt{-39}}{2}\right)=(u)$. We note that $\chi(u)=1$ while $\chi(-u)=-1$, and so take $\tilde{\chi}\left(\mathfrak{p}_{2}, u\right)=1$ and $\tilde{\chi}\left(\mathfrak{p}_{2},-u\right)=\zeta_{8}$ for some primitive 8th root of unity.

Note that twisting $\mathbf{G}$ by a Hilbert character merely changes the embedding. Indeed, if we consider $u^{1 / 4}$ as fixed and write $\mathbf{G}_{j}$ for the Grössencharacter for the embedding with $\zeta_{4}^{j} u^{1 / 4}$ instead of $u^{1 / 4}$, we get $\mathbf{G}_{j}\left(\mathfrak{p}_{2}\right)=\zeta_{4}^{j} u^{1 / 4}=\psi^{j}\left(\mathfrak{p}_{2}\right) u^{1 / 4}=\psi^{j}\left(\mathfrak{p}_{2}\right) \mathbf{G}_{0}\left(\mathfrak{p}_{2}\right)$, and this same calculation passes over to all ideals (via multiplicativity, and triviality of Hilbert characters on principal ideals), and so we get $\mathbf{G}_{j}=\psi^{j} \cdot \mathbf{G}_{0}$.

The computation for the choice of the lift $\tilde{\chi}$ is similar. Upon taking $\tilde{\chi}\left(\mathfrak{p}_{2}, u\right)=\zeta_{4}^{j}$ we get $\mathbf{G}_{\tilde{\chi}}\left(\mathfrak{p}_{2}\right)=\mathbf{T}(u)^{1 / 4} \tilde{\chi}\left(\mathfrak{p}_{2}, u\right)=u^{1 / 4} \zeta_{4}^{j}=\mathbf{G}_{j}\left(\mathfrak{p}_{2}\right)$, with the computation again transferring to all ideals via translation. The independence (up to embeddings) of the choice of a unit with $u$ can be verified by noting that the expressions $\mathbf{T}(u)^{1 / 4} \tilde{\chi}\left(\mathfrak{p}_{2}, u\right)$ and $\mathbf{T}(-u)^{1 / 4} \tilde{\chi}\left(\mathfrak{p}_{2},-u\right)=$ $(-1)^{1 / 4} \mathbf{T}(u)^{1 / 4} \zeta_{8}$ differ by some 4 th root of unity.

We can also note here that while each of the four Hilbert characters has sign +1 in its functional equation ( ince $\psi\left(\mathfrak{d}_{K}\right)=\psi\left(\mathfrak{p}_{3} \mathfrak{p}_{13}\right)=+1$ ), two of the $\mathbf{G} \psi^{j}$ have sign +1 and two have sign -1 . Indeed, using the above formula (4) we have

$$
\epsilon_{\mathbf{G} \psi^{j}}=\epsilon_{\mathbf{G}} \cdot \epsilon_{\psi^{j}} \cdot \tilde{\mathbf{G}}^{-1}(1) \cdot \psi^{-j}\left(\mathfrak{c}_{\mathbf{G}}\right)=\epsilon_{\mathbf{G}} \cdot \psi^{-j}\left(\mathfrak{c}_{\mathbf{G}}\right),
$$

and we note that $\psi\left(\mathfrak{c}_{\mathbf{G}}\right)=-1$ (where here $\mathfrak{c}_{\mathbf{G}}$ is either $\mathfrak{p}_{3}$ or $\mathfrak{p}_{13}$, depending on the choice of $\chi)$.

5.5.1. Caveat. - The above correspondence between Hilbert characters and embeddings needs to be adjusted slightly when $\mathbf{T}\left(u_{i}\right)$ is a $k$ th power for some $k \mid o_{i}$ (with $k>1$ ). For instance, the principal [3,0] Grössencharacter for $K=\mathbf{Q}(\sqrt{-23})$ has $K$ as its value field, and twisting by a nontrivial Hilbert character adjoins $\zeta_{3}$.

\section{Assorted examples}

\subsection{Critical values and elliptic curves. -}

Let $K=\mathbf{Q}(\sqrt{-23})$ and $\mathbf{G}$ be a Grössencharacter of $\infty$-type [2,0] for the trivial modulus. There are three twists of $\mathbf{G}$ corresponding to Hilbert characters (and/or embeddings, as above), and we let $\psi$ be a nontrivial Hilbert character. There are a number of field extensions that arise here. We write $E_{621}$ for the field defined by $\beta^{3}-6 \beta-3=0$, so that $E=K E_{621}$ is the value-field of $\mathbf{G}$ (with a twist by $\mathbf{Q}\left(\zeta_{3}\right)$ when the embedding changes). For the real cubic subfield $H_{-23}$ of the Hilbert class field $H / K$ we write $\alpha^{3}-\alpha-1=0$.

We obtain that the special values at the edge of the critical strip are, up to permutation, approximated as:

$$
\begin{aligned}
L(\mathbf{G}, 2) & \approx 1.06110583266449728309907405960, \\
L(\mathbf{G} \psi, 2) & \approx 1.23819100212426040400794384795 . \\
L\left(\mathbf{G} \psi^{2}, 2\right) & \approx 0.670337208665839403747922477472,
\end{aligned}
$$


We can compute that

$$
L(\mathbf{G}, 2) L(\mathbf{G} \psi, 2) L\left(\mathbf{G} \psi^{2}, 2\right)=\frac{\pi^{3}}{23^{3}} \frac{1}{3 \sqrt{23}} \prod_{i=1}^{22} \Gamma(i / 23)^{\chi(i)},
$$

where $\chi$ is the quadratic Dirichlet character modulo 23 (see [19, III.1.4]), and can note that this $L$-product is also equal to $\frac{\pi^{2}}{5} L\left(\mathbf{G}^{3}, 4\right)$. For the root number, we compute $\mathbf{G}\left(\mathfrak{p}_{23}\right)=-23$ so that $\epsilon_{\mathfrak{p}_{23}}(\mathbf{G})=-1$; when combined with $\epsilon_{\infty}(\mathbf{G})=i^{2-0}=-1$ this implies that $\epsilon_{\mathbf{G}}=+1$, as expected for self-dual $\mathbf{G}$ and even weight.

6.1.1. Ratios of the L-values. - The six pairwise ratios of the above $L$-values lie in the degree 18 field $E_{621} \cdot \mathbf{Q}(\sqrt{69}) \cdot H_{-23}$, and the cubes of these ratios are all conjugate in the degree 6 field $E_{621} \cdot \mathbf{Q}(\sqrt{69})$, which is the Galois closure of the cubic field $E_{621}$, and is also the maximal real subfield of the Galois closure of the value-field. Indeed, the six nontrivial $L\left(\mathbf{G} \psi^{i}, 2\right)^{3} / L\left(\mathbf{G} \psi^{j}, 2\right)^{3}$ ratios are given by the six embeddings of $1 / 541696$ times

$$
(23832 \sqrt{69}+182478) \beta^{2}-(79253 \sqrt{69}+221763) \beta+(-79355 \sqrt{69}+434509) .
$$

Letting $t$ be this element, it has relative norm 1 in $\mathbf{Q}(\sqrt{69})$ we so we can apply Hilbert's Theorem 90 [13, Satz 90, $\S 54$, Capitel XV, p. 272] to a 3 -cycle $\sigma$ of this $S_{3}$-extension and get an element $v$ with $\sigma(v) / v=t$. We can further factor $v=e s$ for some $e \in E_{621}$ and $s \in \mathbf{Q}(\sqrt{69})$, and take

$$
e=\left(365 \beta^{2}+53 \beta+431\right)=-\left(5+\beta-\beta^{2}\right)^{9}\left(2-\beta^{2}\right)^{3} \eta_{1}^{2} \eta_{2}^{3}
$$

(unique up to $\mathbf{Q}$-scaling), where the first two elements have respective norms 2 and 23, and the other two are units $\eta_{1}=-2-\beta$ and $\eta_{2}=1+2 \beta$.

The end result of this is that

$$
\left(L\left(\mathbf{G}_{\iota}, 2\right)^{3}\right)_{\iota} \approx e \otimes 0.00059831771559950518493950 \approx e \otimes z \in E_{621} \otimes \mathbf{R},
$$

where this indicates $L\left(\mathbf{G}_{\iota}, 2\right)^{3} \approx \iota(e) z$ for each embedding $\iota: E_{621} \hookrightarrow \mathbf{R}$. I do not see how to remove the cubing here, even if one passes to value-field $E=K E_{621}$, as no rational multiple of $e$ is a cube; nor do I see any redoing of the computation with different choices that would lead to $e$ having a cube root. Indeed, the six nontrivial $L\left(\mathbf{G} \psi^{i}, 2\right) / L\left(\mathbf{G} \psi^{j}, 2\right)$ ratios really do live in the degree 18 field obtained from adjoining the real subfield $H_{-23}$ of the Hilbert class field to the degree 6 value-field.

6.1.2. Periods from an elliptic curve. - The period $z$ of above corresponds to the period of an elliptic curve $C$ that is defined over the real subfield of the Hilbert class field ([21, Corollary, Theorem 10]), and this curve can be obtained from the $j$-invariant $j\left(\frac{1+\sqrt{-23}}{2}\right)=$ $-5^{3}\left(2+\alpha+\alpha^{2}\right)^{3}(2+3 \alpha)^{3}$ as the curve must have complex multiplication by $K$ (see $[\mathbf{8}, \S 12]$ for more on this). We need to enlarge the places of ramification to include those for $K(j) / \mathbf{Q}(j)$ (see also [18] in this regard), which turns out to be the prime above 23 that is not already ramified in $\mathbf{Q}(j)$. We can get the desired curve by starting with a curve with the given $j$-invariant and then twisting away any ramification at undesired places.

We obtain that $C$ is given by

$$
y^{2}+(1+\alpha) x y+y=x^{3}-x^{2}-\left(17+27 \alpha+12 \alpha^{2}\right) x-\left(45+72 \alpha+61 \alpha^{2}\right),
$$


and that the real period corresponding to the real embedding of $H_{-23}$ is approximately given by $\omega_{1}=0.8174695113021061400156361160297$ - as a check, the "imaginary" period differs by a factor of $\sqrt{23}$ from this, as $(1+\sqrt{-23}) \omega_{1} / 2$. The other periods of $C$ can be approximated as

$$
\omega_{2} \approx 1.84329374387271752388216654 \pm 0.34697676998110995398120 i,
$$

with the lattices being given by $\left[\omega_{2}, \frac{1+\sqrt{-23}}{4} \omega_{2}\right]$ and $\left[\overline{\omega_{2}}, \frac{-1+\sqrt{-23}}{4} \overline{\omega_{2}}\right]$, and these correspond to the binary quadratic forms $2 x^{2} \mp x y+3 y^{2}$. Upon writing the product as $\Omega=\omega_{1} \omega_{2} \bar{\omega}_{2} \approx 2.87595968338354139672$, we can re-compute the above product of $L$-values as $\prod_{i} L\left(\mathbf{G} \psi^{i}, 2\right) \approx \frac{23}{216} \Omega^{2}$.

6.1.3. Relating the periods. - The above calculation computes the period directly for the Grössencharacter given by $\mathbf{G} \circ \mathrm{N}_{H / K}: H \rightarrow K$ and via $[\mathbf{9}, \S 4.7$ (12)] (see also [19, II.1.8.11, II.1.4.5]) we can relate it to $\omega\left(\mathbf{G}^{3}\right)$ via the formula

$$
\frac{\omega\left(\mathbf{G} \circ \mathrm{N}_{H / K}\right)}{\omega\left(\mathbf{G}^{[H: K]}\right)}=\left|\begin{array}{ccc}
1 & 1 & 1 \\
\alpha & \alpha^{\sigma} & (\alpha)^{\sigma^{2}} \\
\alpha^{2} & \left(\alpha^{2}\right)^{\sigma} & \left(\alpha^{2}\right)^{\sigma^{2}}
\end{array}\right|=-\sqrt{-23} \in K,
$$

where we have used the fact that the factor that comes from the field discriminant (see $[\mathbf{1 9}$, II.1.7.12(iv)]) is trivial here (as the field is $\mathbf{Q}$ ). Since the value-field of $\mathbf{G}^{3}$ is $K$ itself, both periods on the left are in $K \otimes \mathbf{C}$.

We can also compute that $L\left(\mathbf{G}^{3}, 4\right)(2 \pi i)^{2} \approx-\frac{5 \cdot 23}{54} \Omega^{2}$, and in this manner can thus obtain $\omega\left(\mathbf{G}^{3}\right)=(a+b \sqrt{-23}) \otimes \Omega^{2} \in K \otimes \mathbf{C}$ for some $a, b \in \mathbf{Q}$ as a direct experimental fact, independent of the above relation to the Shimura periods. Furthermore, we can compute that $L\left(\mathbf{G}^{3}, 5\right)(2 \pi i) \approx \frac{23 \sqrt{-23}}{216} \Omega^{2}$, which shows how the extra factor of $i$ in the $2 \pi i$ intervenes when raised to an odd power.

6.1.4. Reduction in periods. - We now proceed as in $[\mathbf{7}, \S 4.10-12, \S 9]$ to compute the period for $\mathbf{G}$. We let $\sigma \in \operatorname{Gal}(H / K)$ be nontrivial, and take $\mathfrak{a}=\mathfrak{p}_{2}$ as an element with nontrivial image in the class group. This $\mathfrak{p}_{2}$ gives an isogeny $C \rightarrow D$ for some elliptic curve $D$, and by taking $\sigma$ and $\mathfrak{p}_{2}$ properly for Artin reciprocity, we have an isomorphism $D \rightarrow C^{\sigma}$ (where here $C, C^{\sigma}, D$ are taken to mean explicit models). We pullback a Néron differential from $C^{\sigma}$ to $C$ along these maps, and get

$$
\Lambda\left(\mathfrak{p}_{2}\right)=\frac{1}{2 \sqrt{-23}}\left(3+\sqrt{-23}+(1-\sqrt{-23}) \alpha+(7+\sqrt{-23}) \alpha^{2}\right)
$$

in (4.10) of [7]. In (4.11), we thus get an element of relative norm 1 given by

$$
\begin{aligned}
\Phi(\sigma)=\frac{1}{\sqrt{-23}}(-8- & \left.\frac{3+\sqrt{-23}}{2} \beta+2 \beta^{2}\right) \otimes \\
& \otimes \frac{1}{4 \sqrt{-23}}\left(-9+3 \sqrt{-23}+(3-2 \sqrt{-23}) \alpha+(2-2 \sqrt{-23}) \alpha^{2}\right)
\end{aligned}
$$

as an element of $E \otimes_{K} H$. The $E$-element is a cube root of $u_{2}=-(3+\sqrt{-23}) / 2$ and the $H$-element $h$ satisfies $h \sigma(h) \sigma(\sigma(h))=1 / u_{2}$, with $\mathfrak{p}_{2}^{3}=\left(u_{2}\right)$ in $K$.

As in (4.12) of [7] we then apply Hilbert's Theorem 90 to find $\sigma(x) / x=\Phi(\sigma)$ with

$$
23 x=\left(17 \beta^{2}-30 \beta-22\right) \otimes 1+\left(-2 \beta^{2}+13 \beta+8\right) \otimes \alpha+\left(9 \beta^{2}-24 \beta-13\right) \otimes \alpha^{2}
$$


and this is in $E_{621} \otimes H_{-23}$ (and unique up to elements in $E_{621}$ ). This $x$ corresponds to the $[1,0] \infty$-type, and we will square it in the formulæ below to get the desired period for the $[2,0] \infty$-type. We can note ${ }^{(1)}$ that $x^{3}=\left(\eta_{2} / \eta_{1}\right)^{2} \otimes \alpha^{2}$.

We see that Theorem 9.1 of $[\mathbf{7}]$ says $L(\mathbf{G}, 2) /(x \omega)^{2} \in E \otimes 1$ upon choosing the same embedding $H \hookrightarrow \mathbf{C}$ for both $x \in E_{621} \otimes H \hookrightarrow E_{621} \otimes \mathbf{C}$ and the elliptic curve $C$ in the definition of $\omega$, and $[\mathbf{7}, \S 8]$ explains how to reduce the situation to real subfields.

We embed $H_{-23} \hookrightarrow \mathbf{R}$ with $\omega=\omega_{1}$ and $\alpha \approx 1.324717957244746025961$, and so

$$
\left(L\left(\mathbf{G}_{\iota}\right)\right)_{\iota}=\frac{1}{24 \cdot 23^{2}}\left(1767+165 \beta-307 \beta^{2}\right) \otimes\left(x \omega_{1}\right)^{2} \in E_{621} \otimes \mathbf{R}
$$

when embedding both $x$ and $\omega$ into the reals. Finally, we can note that

$$
\left(L\left(\mathbf{G}_{\iota}\right)^{3}\right)_{\iota}=\frac{1}{2^{9} \cdot 3}\left(365 \beta^{2}+53 \beta+431\right) \otimes\left(\alpha^{2} \omega_{1}^{3}\right)^{2} \in E_{621} \otimes \mathbf{R}
$$

when embedding $\alpha$ into the reals, with an additional factor of $-19 \pm 4 \sqrt{-23} \in K$ on the right when using the other embeddings. Indeed, we have that $\alpha^{2} \omega_{1}^{3}=\Omega / 3$ under the real embedding, with $-2 \Omega / 3$ on the right with the others, which relates $\omega\left(\mathbf{G}^{3}\right)$ with $\omega(\mathbf{G})^{3}$.

6.2. A vanishing central symmetric-cube value. - This example is mentioned in $[\mathbf{1 6}$, $\S 4$, Remark 2]. We take $K=\mathbf{Q}(\sqrt{-59})$, which has class number 3, and consider the principal Grössencharacter $\mathbf{G}$ of $\infty$-type $[3,0]$ modulo $\mathfrak{p}_{59}$ (with $\chi$ as the quadratic character). We see that $\mathbf{G}$ will take values in $K$, and indeed the $L$-function of $\mathbf{G}$ is the same as that for a rational weight 4 modular form of level $59^{2}$. The interest of this example is that central value $L(\mathbf{G}, 2)=0$ vanishes even though the sign of the functional equation is even. This special value can be computed exactly as in [16], among other methods (such as modular symbols), so that the vanishing is known. To the best of my knowledge, the conjectural rephrasing as the leading term of the Taylor series $L^{\prime \prime}(\mathbf{G}, 2) \approx 5.752742016791747931531921931$ as an explicit regulator has not yet been achieved, though the works [20] and [3] do construct cycles in Griffiths groups in similar contexts.

We can note that $[\mathbf{2 4}, \S 6.6 .2]$ gives more examples of such "analytic rank 2 motives" (in quadratic twist families corresponding to CM elliptic curves over $\mathbf{Q}$ ), but also indicates (inter alia) that there is no known degree $2 \mathrm{~L}$-function of (motivic) weight greater than 1 whose "analytic rank" is more than 2, with a large amount of data computed in the Grössencharacter case.

6.3. An example over $\mathbf{Q}\left(\zeta_{5}\right)$. — The above examples have all dealt with imaginary quadratic fields. We now give one over a larger field. In order to deal with $\infty$-types in a reasonable manner, we will fix embeddings. We first consider the $\infty$-type $([3,0],[1,2])$ where the first

\footnotetext{
${ }^{(1)}$ This means that $x$ has a square root in this field (note that we have chosen the $E$-scaling to ensure this, so the content here is that the $H$-component is square), but I do not know if this is of import. It could be related to the fact that Shimura's periods can also be used with "half-integral" Grössencharacters in some contexts (for instance, see Remark 1.7(a) of [10] - my understanding is that Shimura's periods extend to the situation of an $\infty$-type such as $\left[\frac{1}{2},-\frac{1}{2}\right]$, though I could be mistaken). Also, it becomes tricky to make refined statements about integrality as the use of Hilbert's theorem 90 gives us the freedom of an element of $E=K(\mathbf{G})$, though see $[\mathbf{7}, \S 10]$ for more in this regard.
} 
pair of (complex conjugate) embeddings sends $\zeta_{5} \rightarrow e^{2 \pi i(1 / 5)}, e^{2 \pi i(4 / 5)}$ and the second to $\zeta_{5} \rightarrow$ $e^{2 \pi i(2 / 5)}, e^{2 \pi i(3 / 5)}$. We shall see that this is rather different from the $([3,0],[2,1]) \infty$-type.

We note $\mathbf{T}\left(\zeta_{5}\right)=\left(e^{2 \pi i(1 / 5)}\right)^{3}\left(e^{2 \pi i(4 / 5)}\right)^{0}\left(e^{2 \pi i(2 / 5)}\right)^{1}\left(e^{2 \pi i(3 / 5)}\right)^{2}=\left(e^{2 \pi i / 5}\right)^{11}$, and so any modulus $\mathcal{I}$ must afford a Dirichlet character of order 5 for the character $\chi_{\mathbf{U}_{K}}^{\mathbf{T}}$ to lift. As above, we are most interested in characters whose ramification is non-disjoint from that of the field, and so we work with $\mathcal{I}=\mathfrak{p}_{5}^{2}$ where $\mathfrak{p}_{5}$ is the totally ramified prime of norm 5 . We note that $\epsilon=1+\zeta_{5}^{2}$ is a unit with $\mathbf{T}(\epsilon)=-e^{2 \pi i / 5}$, and so take $\chi$ of order 10 such that $\chi\left(\zeta_{5}\right)=\left(e^{2 \pi i / 5}\right)^{-1}$ and $\chi(\epsilon)=-\left(e^{2 \pi i / 5}\right)^{-1}$. Since the class group is trivial, we can compute the principal Grössencharacter $\mathbf{G}$ on any ideal directly via principalisation. For instance, we can write

$$
(11) \mathbf{Z}_{K}=\prod_{\sigma}\left(1-\zeta_{5}-\zeta_{5}^{3}\right)^{\sigma}=\left(t_{1}\right)\left(t_{4}\right)\left(t_{2}\right)\left(t_{3}\right)
$$

with $\chi\left(t_{j}\right)=\left(e^{2 \pi i / 5}\right)^{4 j}$ which leads to

$$
\begin{aligned}
& \mathbf{G}\left(t_{1}\right) \approx 8.2745751406263143974426646+35.53211795322830188628510 i, \\
& \mathbf{G}\left(t_{3}\right) \approx 36.225424859373685602557335+4.326499018591243213111138 i,
\end{aligned}
$$

while $\mathbf{G}\left(t_{4}\right)=\overline{\mathbf{G}}\left(t_{1}\right)$ and $\mathbf{G}\left(t_{3}\right)=\overline{\mathbf{G}}\left(t_{2}\right)$.

The functional equation in this case has $\Gamma(s) \Gamma(s-1)$ as the factor for $L_{\infty}(\mathbf{G}, s)$, with $\Lambda(\mathbf{G}, s)=\epsilon_{\mathbf{G}} \Lambda(\overline{\mathbf{G}}, 4-s)$ where $\epsilon_{\mathbf{G}}=\epsilon_{\mathfrak{p}_{5}}=+1$ can be computed via

$$
\sum_{j=1}^{4} \sum_{k=0}^{4} \chi\left(j+k e_{5}\right) e^{2 \pi i \cdot \operatorname{tr}\left(\left(j+k e_{5}\right) / e_{5}^{5}\right)}=5, \text { with }\left(e_{5}\right)^{5}=\left(1-\zeta_{5}\right)^{5}=\mathfrak{p}_{5}^{5}=\mathfrak{d}_{\mathfrak{p}_{5}} \mathfrak{c}_{\mathfrak{p}_{5}}
$$

We compute that the central value is given by

$$
L(\mathbf{G}, 2)=\frac{1}{5^{7 / 2}} \frac{\Gamma(1 / 5)^{3} \Gamma(2 / 5)^{3}}{\Gamma(3 / 5)^{2} \Gamma(4 / 5)^{2}}
$$

where these exponents could perhaps be derived directly from [19, II.4.1].

If we now follow the same calculation with the $([3,0],[2,1]) \infty$-type, we find that $\mathbf{T}\left(\zeta_{5}\right)=$ $\left(e^{2 \pi i / 5}\right)^{3 \cdot 1+4 \cdot 0+2 \cdot 2+3 \cdot 1}=1$.

However we still have $\mathbf{T}(\epsilon)=-1$ and thus there is no Grössencharacter with trivial modulus. Instead we take $\mathcal{I}=\mathfrak{p}_{5}$ and $\chi$ of order 2 with $\chi(\epsilon)=-1$, and can compute that

$$
L(\mathbf{G}, 2)=\frac{\pi}{5^{15 / 4}} \frac{\Gamma(1 / 5)^{7 / 2} \Gamma(3 / 5)^{1 / 2}}{\Gamma(4 / 5)^{7 / 2} \Gamma(2 / 5)^{1 / 2}} .
$$

Again we have $\epsilon_{\mathfrak{p}_{5}}=+1$ via the computation $\sum_{j} \chi(j) e^{2 \pi i \cdot \operatorname{tr}\left(j / e_{5}^{4}\right)}=\sqrt{5}$. The root-number contribution from infinite places is again $i^{3-0} i^{2-1}=+1$.

\section{References}

[1] D. Blasius, On the Critical Values of Hecke L-Series. Ann. Math. 124 (2), no. 1 (1986), $23-63$. Available online from http://www.jstor.org/stable/1971386 
[2] W. Bosma, J. Cannon, C. Playoust, The Magma algebra system. I. The user language. In Computational algebra and number theory, Proceedings of the 1st Magma Conference held at Queen Mary and Westfield College, London, August 23-27, 1993. Edited by J. Cannon and D. Holt, Elsevier Science B.V., Amsterdam (1997), 235-265. Cross-referenced as J. Symbolic Comput. 24 (1997), no. 3-4, 235-265.

Available online from http://magma.maths.usyd.edu.au

[3] J. Buhler, C. Schoen, J. Top, Cycles, L-functions and triple products of elliptic curves. J. Reine Angew. Math. 492 (1997), 93-133.

Available online from http://resolver.sub.uni-goettingen.de/purl?GDZPPN002215160

[4] J. Coates, S. Lichtenbaum, On l-adic zeta functions. Ann. Math. (2) 98 (1973), 498-550. Available online from http://www.jstor.org/stable/1970916

[5] P. Deligne, Valeurs de fonctions $L$ et périodes d'intégrales. (French) [Values of $L$-functions and periods of integrals.] In Automorphic forms, representations, and L-functions, part 2, Proceedings of the Symposium in Pure Mathematics of the American Mathematical Society (Twenty-fifth Summer Research Institute, Corvallis, OR). Edited by A. Borel and W. Casselman. AMS Proc. Symp. Pure Math. XXXIII, no. 2. Amer. Math. Soc., Providence, RI (1979), 313-346. Available online from http: //www . ams.org/online_bks/pspum332

[6] T. Dokchitser, Computing Special Values of Motivic L-functions. Experiment. Math. 13 (2003), no. 2, 137-149. Available online from

http://www . expmath.org/expmath/volumes/13/13.2/Dokchitser.pdf

[7] C. Goldstein, N. Schappacher, Séries d'Eisenstein et fonctions L de courbes elliptiques à multiplication complexe. (French) [Eisenstein series and $L$-functions of elliptic curves with complex multiplication]. J. reine angew. Math. 327 (1981), 184-218.

Available online from http://resolver.sub.uni-goettingen.de/purl?GDZPPN002198800

[8] B. H. Gross, Arithmetic on Elliptic Curves with Complex Multiplication. With an Appendix by B. Mazur. Lect. Notes in Math. 776 (1980), Springer-Verlag, Berlin, iii+95pp. Available online from http://dx.doi.org/10.1007/BFb0096754

[9] G. Harder, N. Schappacher, Special values of Hecke L-functions and Abelian integrals. In Arbeitstagung Bonn 1984, Proceedings of the meeting held by the Max-Planck-Institut für Mathematik, Bonn, June 15-22, 1984. Edited by F. Hirzebruch, J. Schwemer, S. Suter, Lecture Notes in Mathematics 1111 (1984), Springer-Verlag, 17-49.

Available online from http://dx.doi.org/10.1007/BFb0084583

[10] M. Harris, L-functions of $2 \times 2$ Unitary Groups and Factorization of Periods of Hilbert Modular Forms. J. Amer. Math. Soc. 6 (1993), no. 3, 637-719.

Available online from http://www.jstor.org/stable/2152780

[11] E. Hecke, Vorlesungen über die Theorie der algebraischen Zahlen. (German) [Lectures on the Theory of algebraic Numbers]. Akademische Verlagsgesellschaft, Leipzig, 1923.

[12] F. Hess, S. Pauli, M. E. Pohst, Computing the multiplicative group of residue class rings. Math. Comp. 72 (2003), no. 243, 1531-1548. See http://www.jstor.org/stable/4099848

[13] D. Hilbert, Die Theorie der algebraischen Zahlkörper. (German) [The Theory of the algebraic Number fields]. Jahresbericht der Deutschen Mathematiker-Vereinigung [Yearly report of the German Mathematical Society] 4 (1897), 175-546.

Available online from http://resolver.sub.uni-goettingen.de/purl?GDZPPN002115344

[14] A. F. Lavrik, Functional and approximate functional equations for the Dirichlet function. Mat. Zametki, 3:5 (1968), 613-622.

Online at http://mi . mathnet.ru/eng/mz6720

[15] M. Radziejewski, On the distribution of algebraic numbers with prescribed factorization properties, Acta Arith. 116 (2005), 153-171. See the author's webpage at 
http://www.staff .amu.edu.pl/ maciejr/computations/ /multiplicities_WWW/ZetaChiZeros.html

[16] F. Rodriguez Villegas, Square root formulas for central values of Hecke L-series II. Duke Math. J. 72 (1993), no. 2, 431-440.

Available online from http://projecteuclid.org/euclid.dmj/1077289426

[17] D. E. Rohrlich, On the L-functions of canonical Hecke characters of imaginary quadratic fields. Duke Math. J. 47 (1980), no. 3, 547-557.

Available online from http://projecteuclid.org/euclid.dmj/1077314180

[18] D. E. Rohrlich, Elliptic curves with good reduction everywhere. J. London Math. Soc. (2) 25 (1982), no. 2, 216-222.

Available online from http://jlms.oxfordjournals.org/cgi/reprint/s2-25/2/216.pdf

[19] N. Schappacher, Periods of Hecke characters. Lecture Notes in Mathematics, 1301, SpringerVerlag, Berlin, 1988. xvi+160 pp.

Available online from http://dx.doi.org/10.1007/BFb0082094

[20] C. Schoen, Complex multiplication cycles and a conjecture of Beilinson and Bloch. Trans. Amer. Math. Soc. 339 (1993), no. 1, 87-115.

Available online from http://dx.doi.org/10.2307/2154210

[21] G. Shimura, On the zeta-function of an abelian variety with complex multiplication. Ann. of Math.

(2) 94 (1971), 504-533. See http://www . jstor.org/stable/1970768

[22] C. L. Siegel, Berechnung von Zetafunktionen an ganzzahligen Stellen. (German) [Calculation of Zeta functions at integral Points]. Nachr. Akad. Wiss. Göttingen Math. Phys. Kl. II 10 (1969), 87-102.

[23] J. T. Tate, Fourier analysis in number fields and Hecke's zeta-functions, Ph.D. Thesis, Princeton Univ., Princeton, N.J., 1950, reprinted in Algebraic number theory: proceedings of an instructional conference organized by the London Mathematical Society (a NATO Advanced Study Institute) with the support of the International Mathematical Union, edited by J. W. S. Cassels and A. Fröhlich, Academic Press, London; Thompson Book Co., Inc., Washington, D.C., 1967, xviii+366 pp.

[24] M. Watkins, Some Heuristics about Elliptic Curves. Experiment. Math. 17 (2008), no. 1, 105-125.

22 mai 2010

Mark Watkins, Magma Computer Algebra Group, School of Mathematics and Statistics, Carslaw Building F07, University of Sydney, NSW 2006, AUSTRALIA • E-mail : watkins@maths.usyd.edu.au 